\title{
An Apriori-Based Algorithm for Mining Frequent Substructures from Graph Data
}

\author{
Akihiro Inokuchi`, Takashi Washio, and Hiroshi Motoda \\ I.S.I.R., Osaka University \\ 8-1, Mihogaoka, Ibarakishi, Osaka, 567-0047, Japan \\ Phone: +81-6-6879-8541 Fax: +81-6-6879-8544 \\ washio@sanken.osaka-u.ac.jp
}

\begin{abstract}
This paper proposes a novel approach named AGM to efficiently mine the association rules among the frequently appearing substructures in a given graph data set. A graph transaction is represented by an adjacency matrix, and the frequent patterns appearing in the matrices are mined through the extended algorithm of the basket analysis. Its performance has been evaluated for the artificial simulation data and the carcinogenesis data of Oxford University and NTP. Its high efficiency has been confirmed for the size of a real-world problem....
\end{abstract}

\section{Introduction}

Mining knowledge from structured data is a major research topic in recent data mining study. "Graph structure" is one of the representatives of the structured data since it frequently appears in real-world data such as web links and chemical compounds structures. In the field of chemistry, CASE and MultiCASE systems have been often used to discover characteristic substructures of chemical compounds 8, 9 . Though these systems can efficiently find the substructures, the class of the substructures is limited to the no-branching atom sequences. Wang and Liu proposed the mining of wider class of substructures which are subtrees called schemas 14 . Though the proposed algorithm is very efficient to mine frequent schemas from massive data, the mining patterns are still limited to acyclic graphs. To mine characteristic patterns having general graph structures, the propositional classification techniques, e.g., C4.5, the regression tree techniques, e.g., M5, and the inductive logic programming (ILP) techniques have been applied in the carcinogenesis predictions of chemical compounds [10, 7]. However, these approaches can discover only limited types of characteristic substructures, because the graph structures must be pre-characterized by some specific features and/or ground instances of predicates.

Recently, a technique to mine the frequent substructures characterizing the carcinogenesis of chemical compounds has been proposed without requiring any conversion of substructures to specific features by Dehaspe et al. [3. They used

\footnotetext{
* Currently beeing in Tokyo Research Institute, IBM, 1623-14 Shimotsuruma, Yamatoshi, Kanagawa, 242-8502, Japan.
} 
the ILP framework combined with levelwise search to minimize the access frequency to the database [11. Since the efficiency achieved by this approach is much better than the former ILP approaches, some new discovery of substructures characterizing carcinogenesis was expected. However, the full search space was still so large that the search had to be limited within the 6th level where the substructures are represented with 6 predicates at maximum, and they reported that significant substructures have not been obtained within the search level. Some other researches have also developed the techniques to mine the frequent substructures in graph data. The graph-based induction (GBI) is an approach to seek the frequent patterns by iteratively chunking the vertex pairs that frequently appear 12. SUBDUE is another approach to seek the characteristic graph patterns to efficiently compress the original graph in terms of MDL principle 2. These approaches do not face the severe computational complexity. However, they may miss some significant patterns, since their search strategies are greedy.

Though the task tackled by these works involves the problem of deciding graph isomorphism which is known to be NP, each work mines some characteristic graph substructures by introducing the limitations on the search space and/or the class of substructures. The objective of this paper is 1) to propose a novel approach named as "Apriori-based Graph Mining", AGM for short, to mine the frequent substructures and the association rules from the general class of graph structured data in a more efficient manner than the preceding work, and 2) to assess the performance of the approach for the artificially simulated data and also for the carcinogenesis data of Oxford University and National Toxicological Program (NTP) [13.

\section{Principle of Mining Graph Substructures}

The methods studied in the mathematical graph isomorphism problem are not directly applicable to our case, because the methods are only to check if the two given graphs are isomorphic 4 . We introduce the mathematical graph representation of "adjacency matrix" and to combine it with an efficient levelwise search of the frequent canonical matrix code [5. The levelwise search is based on the extension of the Apriori algorithm of the basket analysis 11.

\subsection{Representation of Graph Structures}

A graph in which the vertices and edges have labels is mathematically defined as follows.

Definition 1 (Graph having Labels) Given a set of vertices $V(G)=\left\{v_{1}, v_{2}\right.$, $\left.\ldots, v_{k}\right\}$, a set of edges connecting some vertex pairs in $V(G) ; E(G)=\left\{e_{h}=\right.$ $\left.\left(v_{i}, v_{j}\right) \mid v_{i}, v_{j} \in V(G)\right\}$, a set of vertex labels $L(V(G))=\left\{l b\left(v_{i}\right) \mid \forall v_{i} \in V(G)\right\}$ and a set of edge labels $L(E(G))=\left\{l b\left(e_{h}\right) \mid \forall e_{h} \in E(G)\right\}$, then a graph $G$ is represented as

$$
G=(V(G), E(G), L(V(G)), L(E(G))) .
$$


This graph $G$ is represented by an adjacency matrix $X$ which is a very well known representation in mathematical graph theory [4]. This transformation from $G$ to $X$ does not require much computational effort.

Definition 2 (Adjacency Matrix) Given a graph $G=V(G), E(G), L(V(G)$ ), $L(E(G)))$, the adjacency matrix $X$ has the following $(i, j)$-element, $x_{i j}$,

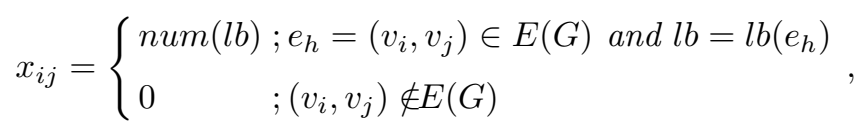

where num $(l b)$ is an integer arbitrarily assigned to a label value lb. Moreover, a number num(lb) is assigned to the $i$-th low ( $i$-th column) of the matrix where $v_{i} \in V(G)$ and $l b=l b\left(v_{i}\right)$.

Definition 3 (Size of a Graph) The "size" of a graph $G$ is the number of vertices in $V(G)$, i.e., $k$ in Definition 1.

Definition 4 (Graph Transaction and Graph Data) A graph $G=(V(G)$, $E(G), L(V(G)), L(E(G)))$ is a transaction, and graph data $G D$ is a set of the transactions, where $G D=\left\{G_{1}, G_{2}, \ldots, G_{n}\right\}$.

Each element of an adjacency matrix in the standard definition is either ' 0 ' or ' 1 ', whereas each element in Definition 2 can have the number of an edge label. This extended notion of the adjacency matrix gives a compact representation of a graph having labeled edges, and enables an efficient coding of the graph as shown later.

The representation of the adjacency matrix depends on the assignment of each vertex to the $i$-th row ( $i$-th column). To reduce the variants of the representations and increase the efficiency of the code matching described later, the vertices are sorted according to the numbers of their labels. The adjacency matrix of a graph whose size is $k$ is noted as $X_{k}$, and the graph as $G\left(X_{k}\right)$.

Definition 5 (Vertex-sorted Adjacency Matrix) The adjacency matrix $X_{k}$ of the graph $G\left(X_{k}\right)$ is vertex-sorted if

$$
\operatorname{num}\left(l b\left(v_{i}\right)\right) \leq \operatorname{num}\left(l b\left(v_{i+1}\right)\right) \text { for } i=1,2, \ldots, k-1 .
$$

In the standard basket analysis, items within an itemset are kept in lexicographic order [1]. This enables an efficient control of the generation of candidate itemsets. However, the vertex-sorted adjacency matrices do not have such lexicographic order. Thus, a coding method of the adjacency matrices need to be introduced.

Definition 6 (Code of Adjacency Matrix) In case of an undirected graph, the code code $\left(X_{k}\right)$ of a vertex-sorted adjacency matrix $X_{k}$;

$$
X_{k}=\left(\begin{array}{ccccc}
x_{1,1} & x_{1,2} & x_{1,3} & \cdots & x_{1, k} \\
x_{2,1} & x_{2,2} & x_{2,3} & \cdots & x_{2, k} \\
x_{3,1} & x_{3,2} & x_{3,3} & \cdots & x_{3, k} \\
\vdots & \vdots & \vdots & \ddots & \vdots \\
x_{k, 1} & x_{k, 2} & x_{k, 3} & \cdots & x_{k, k}
\end{array}\right),
$$


is defined as

$$
\operatorname{code}\left(X_{k}\right)=x_{1,1} x_{1,2} x_{2,2} x_{1,3} x_{2,3} x_{3,3} x_{1,4} \cdots x_{k-1, k} x_{k, k},
$$

where the digits are obtained by scanning the elements along the columns at the upper triangular part of $X_{k}$. In case of a directed graph, it is defined as

$$
\operatorname{code}\left(X_{k}\right)=x_{1,1} x_{1,2} x_{2,1} x_{2,2} x_{1,3} x_{3,1} x_{2,3} x_{3,2} \cdots x_{k-1, k} x_{k, k-1} x_{k, k},
$$

where the digits are obtained similarly to the undirected case, but the diagonally symmetric element $x_{j i}$ is added after each $x_{i j}$ when $i \neq j$.

The method proposed in this paper discovers substructures frequently appearing in the graph transaction data $G D$. The rigorous definition of the substructure is given as follows.

Definition 7 (Induced Subraph) Given a graph $G=(V(G), E(G), L(V(G))$, $L(E(G)))$, an induced subgraph of $G, G_{s}=\left(V\left(G_{s}\right), E\left(G_{s}\right), L\left(V\left(G_{s}\right)\right), L\left(E\left(G_{s}\right)\right)\right)$, is a graph satisfying the following conditions.

$$
\begin{gathered}
V\left(G_{s}\right) \subset V(G), E\left(G_{s}\right) \subset E(G), \\
\forall u, v \in V\left(G_{s}\right),(u, v) \in E\left(G_{s}\right) \Leftrightarrow(u, v) \in E(G) .
\end{gathered}
$$

When $G_{s}$ is an induced subgraph of $G$, it is denoted as $G_{s} \subset G$.

\subsection{Algorithm of AGM}

Candidate Generation. The two indices which are identical to the definitions of "support" and "confidence" in the basket analysis are introduced.

Definition 8 (Support and Confidence) Given a graph $G_{s}$, the support of $G_{s}$ is defined as

$$
\sup \left(G_{s}\right)=\frac{\text { number of graph transactions } G \text { where } G_{s} \subset G \in G D}{\text { total number of graph transactions } G \in G D} .
$$

Given two induced subgraphs $G_{b}$ and $G_{h}$, the confidence of the association rule $G_{b} \Rightarrow G_{h}$ is defined as

$$
\operatorname{conf}\left(G_{b} \Rightarrow G_{h}\right)=\frac{\text { number of graphs } G \text { where } G_{b} \cup G_{h} \subset G \in G D}{\text { number of graphs } G \text { where } G_{b} \subset G \in G D} .
$$

If the value of $\sup \left(G_{s}\right)$ is more than a threshold value minsup, $G_{s}$ is called as a "frequent induced subgraph".

Similarly to the Apriori algorithm, the candidate generation of the frequent induced subgraph is made by the levelwise search in terms of the size of the subgraph. Let $X_{k}$ and $Y_{k}$ be vertex-sorted adjacency matrices of two frequent induced graphs $G\left(X_{k}\right)$ and $G\left(Y_{k}\right)$ of size $k$. If both $G\left(X_{k}\right)$ and $G\left(Y_{k}\right)$ have equal 
elements of the matrices except for the elements of the $k$-th row and the $k$-th column, then they are joined to generate $Z_{k+1}$.

$$
\begin{gathered}
X_{k}=\left(\begin{array}{cc}
X_{k-1} & \boldsymbol{x}_{1} \\
\boldsymbol{x}_{2}^{T} & x_{k k}
\end{array}\right), Y_{k}=\left(\begin{array}{cc}
X_{k-1} & \boldsymbol{y}_{1} \\
\boldsymbol{y}_{2}^{T} & y_{k k}
\end{array}\right) \\
Z_{k+1}=\left(\begin{array}{ccc}
X_{k-1} & \boldsymbol{x}_{1} & \boldsymbol{y}_{1} \\
\boldsymbol{x}_{2}^{T} & x_{k k} & z_{k, k+1} \\
\boldsymbol{y}_{2}^{T} & z_{k+1, k} & y_{k k}
\end{array}\right)=\left(\begin{array}{cc|c}
X_{k} & \boldsymbol{y}_{1} \\
& z_{k, k+1} \\
\hline \boldsymbol{y}_{2}^{T} & z_{k+1, k} & y_{k k}
\end{array}\right),
\end{gathered}
$$

where $X_{k-1}$ is the adjacency matrix representing the graph whose size is $k-1, \boldsymbol{x}_{i}$ and $\boldsymbol{y}_{i}(i=1,2)$ are $(k-1) \times 1$ column vectors. $X_{k}$ is called the "first matrix" and $Y_{k}$ the "second matrix". The following relations hold among the vertex-sorted adjacency matrices $X_{k}, Y_{k}$ and $Z_{k+1}$.

$$
\begin{gathered}
l b\left(v_{i} ; v_{i} \in V\left(G\left(X_{k}\right)\right)=l b\left(v_{i} ; v_{i} \in V\left(G\left(Y_{k}\right)\right)=l b\left(v_{i} ; v_{i} \in V\left(G\left(Z_{k+1}\right)\right)\right),\right.\right. \\
l b\left(v_{i} ; v_{i} \in V\left(G\left(X_{k}\right)\right) \leq l b\left(v_{i+1} ; v_{i+1} \in V\left(G\left(X_{k}\right)\right),\right.\right. \\
l b\left(v_{k} ; v_{k} \in V\left(G\left(X_{k}\right)\right)=l b\left(v_{k} ; v_{k} \in V\left(G\left(Z_{k+1}\right)\right),\right.\right. \\
l b\left(v_{k} ; v_{k} \in V\left(G\left(Y_{k}\right)\right)=l b\left(v_{k+1} ; v_{k+1} \in V\left(G\left(Z_{k+1}\right)\right),\right.\right. \\
l b\left(v_{k} ; v_{k} \in V\left(G\left(X_{k}\right)\right) \leq l b\left(v_{k} ; v_{k} \in V\left(G\left(Y_{k}\right)\right) .\right.\right.
\end{gathered}
$$

Here, $i=1, \cdots, k-1 . z_{k, k+1}$ and $z_{k+1, k}$ are not determined by $X_{k}$ and $Y_{k}$. Each can take every integer value num $(l b)$ corresponding to each edge label $l b$ or 0 corresponding to the case that no edge exists between $v_{k}$ and $v_{k+1}$. In case of an undirected graph, $z_{k, k+1}$ and $z_{k+1, k}$ must have an identical value. This join procedure of $X_{k}$ and $Y_{k}$ creates multiple $Z_{k+1} \mathrm{~s}$ for all possible value pairs of $z_{k, k+1}$ and $z_{k+1, k}$. Note that when the labels of the $k$-th vertices $v_{k}$ of $G\left(X_{k}\right)$ and $G\left(Y_{k}\right)$ are the same, exchanging $X_{k}$ and $Y_{k}$ (i.e., taking $Y_{k}$ as the first matrix and $X_{k}$ as the second matrix), produces redundant adjacent matrices. In order to avoid this redundant generation, the two adjacency matrices are joined only when Eq.(3) is satisfied. The vertex-sorted adjacency matrix generated under this condition is called a "normal form".

$$
\text { code(the first matrix) } \leq \text { code(the second matrix) }
$$

In the standard basket analysis, the $(k+1)$-itemset becomes a candidate frequent itemset only when all the $k$-sub-itemsets are confirmed to be frequent itemsets. Similarly, the graph $G$ of size $k+1$ is a candidate of frequent induced subgraphs only when all adjacency matrices generated by removing from the graph $G$ the $i$-th vertex $v_{i}(1 \leq i \leq k+1)$ and all its connected links are confirmed to be frequent induced subgraphs of the size $k$. As this algorithm generates only adjacency matrices of the normal form in the earlier (smaller) $k$-levels, if the adjacency matrix of the graph generated by removing the $i$-th vertex $v_{i}$ is non-normal form, it must be transformed to a normal form to check if it matches one of the normal form matrices found earlier. An adjacency matrix $X_{k}$ of a non-normal form is transformed into a normal form $X_{k}^{\prime}$ by reconstructing the 
matrix structure in a bottom up manner. First, an adjacency matrix of the size $1 \times 1$ is set for each vertex $v_{i} \in G\left(X_{k}\right)$. Then, the pair of the matrices for the vertices $v_{i}, v_{j} \in G\left(X_{k}\right)$ satisfying the constraints of Eq.(2) and (31) are joined by the operation of Eq.(1). At this time, the values of the elements for $\left(v_{i}, v_{j}\right)$ and $\left(v_{j}, v_{i}\right)$ in the original $X_{k}$ are substituted to the non-diagonal elements $z_{1,2}$ and $z_{2,1}$ respectively to reconstruct the structure of $G\left(X_{k}\right)$. Subsequently, the pair of the obtained $2 \times 2$ matrices are further joined according to the constraints of Eq.(1), (2) and (3). The values of the elements $z_{2,3}$ and $z_{3,2}$ are determined from $X_{k}$ in the similar manner. This procedure is repeated until a $k \times k$ matrix $X_{k}^{\prime}$ is obtained. Because $X_{k}^{\prime}$ precisely reflects the structure of $G\left(X_{k}\right)$, and is constructed by following the constraints, $X_{k}^{\prime}$ is a normal form of $X_{k}$. This reconstruction is called "normalization". In the intermediate levels, the normal forms of all induced subgraphs of $G\left(X_{k}\right)$ can be derived. This feature of the normalization is used in the frequency calculation explained latter. The normalization consists of the set of permutations of the rows and the columns of the original matrix $X_{k}$. Thus $X_{k}^{\prime}$ has the relation of $X_{k}^{\prime}=\left(T_{k}\right)^{T} X_{k} T_{k}$ where $T_{k}$ is the transformation matrix. The details of the normalization can be found in [6].

Canonical From. After all candidate induced subgraphs are derived, the support value of each candidate is counted in the database. However, the normal form representation is in general not unique for a graph. For instance, the following two matrices which are both normal forms represent an identical undirected graph with a unique link label.

$$
X_{3}=\left(\begin{array}{lll}
0 & 0 & 1 \\
0 & 0 & 1 \\
1 & 1 & 0
\end{array}\right), Y_{3}=\left(\begin{array}{lll}
0 & 1 & 1 \\
1 & 0 & 0 \\
1 & 0 & 0
\end{array}\right)
$$

If the support value is counted for each representation independently, it has to be summed up to obtain the correct support value for the corresponding graph. To perform this summation efficiently, all normal forms for an identical induced subgraph must be indexed. For this purpose, canonical form is defined for normal forms of adjacency matrices representing an identical induced subgraph, and an efficient method to index each normal form to its canonical form is introduced.

Definition 9 (Canonical Form) Given a set $N F(G)$ of all normal forms of adjacency matrices representing an identical graph $G$, its canonical form $X_{c}$ is defined as $X$ having the minimum code number in $N F(G)$, i.e.,

$$
X_{c}=\arg \min _{X \in N F(G)} \operatorname{code}(X) .
$$

We assume that all the transformation matrices $S_{k-1}$ to the canonical form from the normal forms of every frequent induced subgraph of size $k-1$ are known. Let $X_{k-1}^{m}$ be the matrix obtained by removing the $m$-th vertex $v_{m}(1 \leq m \leq k)$ from $G\left(X_{k}\right) . X_{k-1}^{m}$ is transformed to one of its normal forms, $X_{k-1}^{\prime m}$, by the aforementioned normalization, and thus its transformation matrix $T_{k-1}^{m}$ is known. Furthermore, let $S_{k-1}$ of $X_{k-1}^{\prime m}$ be $S_{k-1}^{m}$, then the transformed canonical form is 
represented by $\left(T_{k-1}^{m} S_{k-1}^{m}\right)^{T} X_{k-1}^{m} T_{k-1}^{m} S_{k-1}^{m}$. The canonical form $X_{c k}$ of $X_{k}$ and the matrices $S_{k}^{m}, T_{k}^{m}$ to transform $X_{k}$ to $X_{c k}$ are obtained from $S_{k-1}^{m}, T_{k-1}^{m}$ by the following expressions. The detailed proof of this transformation can be found in 6].

$$
\begin{gathered}
s_{i j}=\left\{\begin{array}{l}
s_{i j}^{m} 0 \leq i \leq k-1 \text { and } 0 \leq j \leq k-1, \\
1 \quad i=k \text { and } j=k, \\
0 \quad \text { otherwise, }
\end{array}\right. \\
t_{i j}= \begin{cases}t_{i j}^{m} & i<m \text { and } j \neq k, \\
t_{i-1, j}^{m} & i>m \text { and } j \neq k, \\
1 & i=m \text { and } j=k, \\
0 & \text { otherwise, }\end{cases} \\
X_{c k}=\arg \min _{m=1, \cdots, k} \operatorname{code}\left(\left(T_{k}^{m} S_{k}^{m}\right)^{T} X_{k}\left(T_{k}^{m} S_{k}^{m}\right)\right),
\end{gathered}
$$

where $s_{i j}, s_{i j}^{m}, t_{i j}$ and $t_{i j}^{m}$ are the elements of matrix $S_{k}^{m}, S_{k-1}^{m}, T_{k}^{m}$ and $T_{k-1}^{m}$ respectively. $T_{k}^{m} S_{k}^{m}$ which minimize the code is $S_{k}$ of $X_{k}$.

Frequency Calculation. Frequency of each candidate induced subgraph is counted by scanning the database after generating all the candidates of frequent induced subgraphs and obtaining their canonical forms. Every transaction graph $G$ in the database can be represented by an adjacency matrix $X_{k}$, but it may not be a normal form in most cases. Since the candidates of frequent induced subgraphs are normal forms, the normalization must be applied to $X_{k}$ of each transaction $G$ to check if the candidates are contained in $G$. As previously described, the procedure of the normalization of $X_{k}$ can derive the normal form of every induced subgraph of $G$ in the intermediate levels. Thus, the frequency of each candidate is counted based on all normal forms of the induced subgraphs of $G$. When the value of the count exceeds the threshold minsup, the subgraph is a frequent induced subgraph. Once all frequent induced subgraphs are found, the association rules among them whose confidence values are more than a given confidence threshold are enumerated by using the algorithm similar to the standard basket analysis.

\section{Performance Evaluation}

The performance of the proposed AGM was examined using an artificial graph transaction data. The machine used is a PC with $400 \mathrm{MHz} \mathrm{CPU}$ and $128 \mathrm{MB}$ main memory. The size of each transaction is determined by the gaussian distribution with the average $|T|$ and the standard deviation 1 . The vertex labels are randomly determined with equal probability. The edges are attached randomly with the probability of $p$. $L$ basic patterns of the average size $|I|$ are generated, and one of them is randomly overlaid on each transaction. The two groups of the 
test data, one for the directed graph and the other for the undirected graph, are prepared. The direction of the edges are given randomly in the former group.

Figures 1, 2, 3 and 4 show the results of computation time for different number of transactions, number of vertex labels, minimum support threshold and average transaction size for both directed and undirected graphs, respectively. In every parameter setting, the required computational time and the number of the discovered frequent induced subgraphs are less in the case of directed graph. Because the number of possible subgraph patterns is larger due to existence of edge direction, the frequency of each subgraph pattern is smaller. This also reduces the required computation for search. In short summary, the proposed algorithm does not show intractable computational complexity except the cases for graphs of large size in the database.

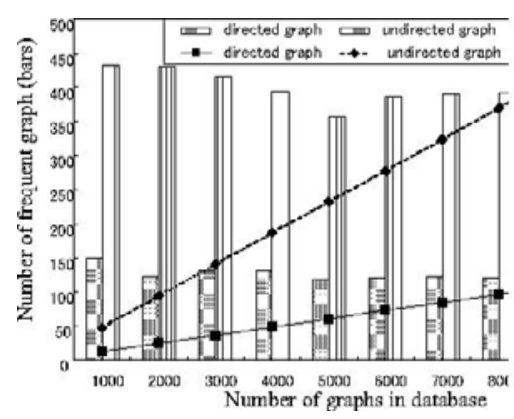

Fig. 1. Complexity v.s. number of transactions.

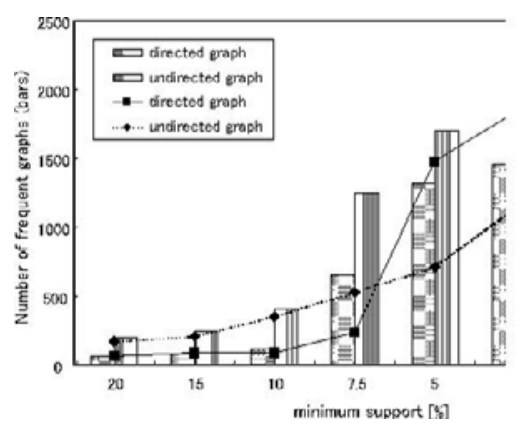

Fig. 3. Complexity v.s. minimum support.

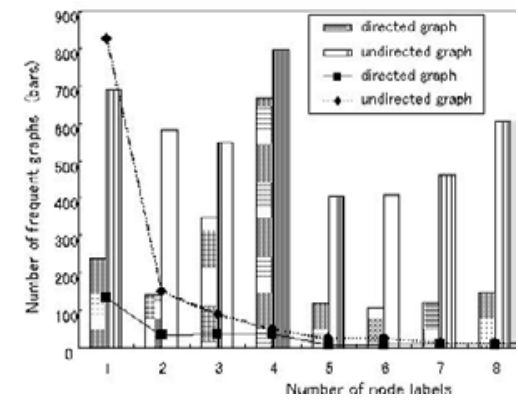

Fig. 2. Complexity v.s. number of vertex labels.

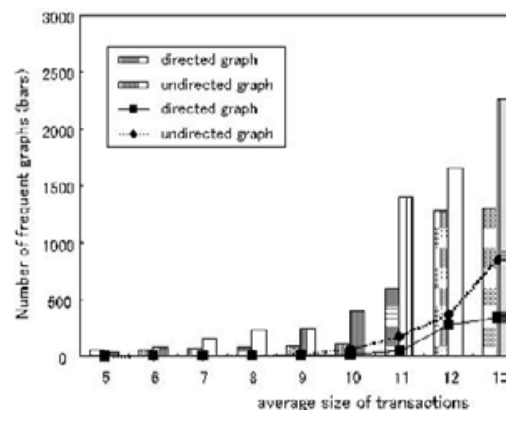

Fig. 4. Complexity v.s. transaction size.

\section{Application to Chemical Analysis}

AGM was applied to chemical carcinogenesis analysis which is a challenge topic proposed in IJCAI-97 by Srinisavan et al. [13]. The task is to find structures typical to carcinogen of organic chlorides. The objective data were obtained from the website of National Toxicology Program (NTP) and Oxford University. Totally, the 300 compounds were selected for the analysis, of which 185 compounds 
Table 1. Results for three minsup values.

\begin{tabular}{|c|cc|cc|cc|}
\hline & \multicolumn{3}{|c|}{ minsup $=20 \%$} & minsup $=15 \%$ & \multicolumn{2}{c|}{ minsup $=10 \%$} \\
\hline L & NOC & NOFS & NOC & NOFS & NOC & NOFS \\
\hline 1 & 24 & 7 & 24 & 8 & 24 & 10 \\
2 & 280 & 62 & 360 & 67 & 550 & 108 \\
3 & 2277 & 477 & 2525 & 640 & 4558 & 964 \\
4 & 6223 & 2178 & 9709 & 3333 & 18268 & 5912 \\
5 & 9767 & 4806 & 18740 & 9372 & 40744 & 19568 \\
6 & 6899 & 4726 & 19813 & 13479 & 56179 & 37219 \\
7 & 2655 & 2179 & 11989 & 9499 & 52082 & 41639 \\
8 & 668 & 655 & 4347 & 4019 & 33208 & 29817 \\
9 & 118 & 118 & 1212 & 1199 & 15618 & 15242 \\
10 & 7 & 7 & 220 & 220 & 5739 & 5725 \\
11 & - & - & 21 & 21 & 1455 & 1455 \\
12 & - & - & 1 & 1 & 23 & 23 \\
13 & - & - & - & - & 15 & 15 \\
\hline Total & 28918 & 15215 & 68961 & 41858 & 228663 & 157897 \\
\hline
\end{tabular}

L:level(number of vertices included in frequent subgraph)

NOC:number of candidates, NOFS:number of frequent graphs

have positive carcinogenesis and the rests are negative. Thus, the fraction of the carcinogenic compounds is $61.7 \%$. The types of atoms involved in the compounds are $\mathrm{C}, \mathrm{H}, \mathrm{O}, \mathrm{Cl}, \mathrm{F}, \mathrm{S}$ and some cations, and the types of bonds are single, double, aromatic and cation bonds. Each transaction data were preprocessed to add artificial edges from each vertex to every other vertex that is within the distance of 6 edges. Each added edge has a label to indicate the distance between the two vertices that are connected by the edge. This enables us to mine the frequent cooccurrence of some specific structures at a specific distance within 6 . The distance limit of 6 was determined based on the chemical insights that the influence of an atom does not usually propagate along the path more than 6 bonds in molecules of moderate sizes. Furthermore, an isolated vertex labeled by the carcinogenesis class of the compound, i.e., "class vertex", is added to each chemical structure graph.

The analysis was made on the same PC described in the previous section. Table 1 shows the number of the candidate induced subgraphs (NOC) and that of the discovered frequent induced subgraphs (NOF) for each level of the search, i.e., the size of the induced subgraphs. In each minsup case, all frequent induced subgraphs were exhaustively discovered. The computation time required to complete the search was far longer for the minsup of smaller value, and was almost 8 days for $10 \%$, while it was only about 40 minutes for $20 \%$. The size of the largest frequent induced subgraph discovered in the case of $10 \%$ was 13 .

In Figure 5, the confidence deviation $\Delta$ of an association rule $G_{b} \Rightarrow G_{h}$ is given as follows.

$$
\Delta= \begin{cases}\operatorname{conf}\left(G_{b} \Rightarrow G_{h}\right)-f r_{p} & \text { if } G_{h} \text { contains a positive class vertex. } \\ \operatorname{conf}\left(G_{b} \Rightarrow G_{h}\right)-f r_{n} & \text { if } G_{h} \text { contains a negative class vertex. }\end{cases}
$$


Here, $f r_{p}$ is the fraction of positive compounds in the data, i.e., $61.7 \%$ in this case, and $f r_{n}$ is that of negative compounds, i.e., $38.3 \%(=100 \%-61.7 \%)$. The cover rate $C R$ of a set of association rules is the fraction of chemical compounds whose classes are derived by applying the rule set to the data. Given a value of $\Delta_{t h}$, a set of association rules each having $\Delta$ more than the $\Delta_{t h}$ is defined, and $C R$ of the rule set is calculated. As shown in Figure 5, the rule set derived for the $10 \%$ threshold contains some rules having significant confidence. Accordingly, the exhaustive search for low support threshold is considered to be very effective to mine valuable rules.

$$
\begin{gathered}
\mathrm{S}=>\text { Negative } \\
\text { sup }=10.3 \%, \text { conf }=70.5 \%, \Delta=32.2 \%
\end{gathered}
$$

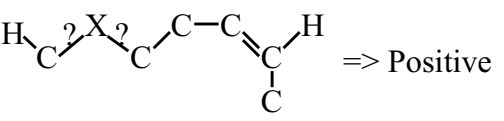

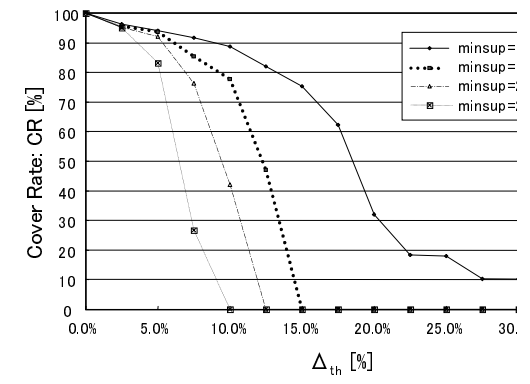

Fig. 5. Relation of $\Delta_{t h}$ and $C R$. $\sup =10.3 \%$, conf $=77.5 \%, \Delta=15.8 \%$

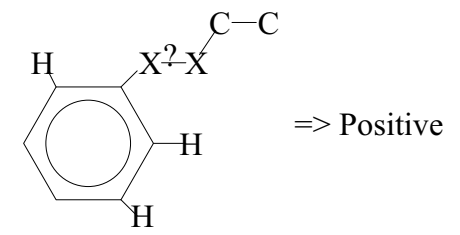

$\sup =10.3 \%$. conf $=73.8 \%, \Delta=12.1 \%$

Figure 6 shows some association rules obtained for the carcinogenesis class under the support threshold $10 \%$. The first rule is very simple, but indicates that a sulfur atom plays an important role to suppress the carcinogenesis. In the second rule, the symbol $X$ of a vertex and ? of an edge indicate that their labels are arbitrary. The third is an example of a less significant but more complex substructure involving a benzene ring. This is consistent with the chemical knowledge that benzene rings frequently have the positive carcinogenesis.

\section{Discussion and Conclusion}

The largest graphs of the chemical compound discovered by AGM have the size of 13 atoms. In contrast, the approach of ILP in conjunction with a levelwise search proposed by Dehaspe et al. could mine the substructure consisting of 6 predicates at maximum equivalent to the size of a molecule consisting of only 3 atoms or so [3]. This fact shows the practical efficiency of AGM for real world problems. Further investigation on the computational efficiency of AGM in terms of the theoretical aspect remains for the future study.

In conclusion, a novel approach has been developed that can efficiently mine frequently appearing induced subgraphs in a given graph data set and the association rules among the frequent induced subgraphs. Its performance has been 
evaluated for both the artificial simulation data and the real world chemical carcinogenesis data. The powerful performance of this approach under some practical conditions has been confirmed through these evaluations.

Acknowledgement. The authors wish to thank Prof. Takashi Okada in Center for Information \& Media Studies, Kwansei Gakuin University for providing us with the chemical compound data and his expertise in the chemistry domain.

\section{References}

1. Agrawal, R. and Srikant, R. 1994. Fast algorithms for mining association rules. In Proc. of the 20th VLDB Conference, pp.487-499.

2. Cook, D.J. and Holder, L.B. 1994. Substructure Discovery Using Minimum Description Length and Background Knowledge, Journal of Artificial Intelligence Research, Vol.1, pp.231-255.

3. Dehaspe, L., Toivonen, H. and King, R.D. 1998. Finding frequent substructures in chemical compounds. In Proceedings of the Fourth International Conference on Knowledge Discovery and Data Mining (KDD-98), pp.30-36.

4. Fortin, S. 1996. The graph isomorphism problem. Technical Report 96-20, University of Alberta, Edomonton, Alberta, Canada.

5. Inokuchi, A., Washio, T. and Motoda, H. 1999. Derivation of the topology structure from massive graph data. Discovery Science: Proceedings of the Second International Conference, DS'99, pp.330-332.

6. Inokuchi, A. 2000. The study on a fast mining method from massive graph structure data. Master thesis (in Japanese), I.S.I.R., Osaka Univ.

7. King, R., Muggleton, S., Srinivasan, A. and Sternberg, M. 1996. Structure-activity relationships derived by machine learning; The use of atoms and their bond connectives to predict mutagenicity by inductive logic programming. In Proceedings of the National Academy of Sciences, Vol.93, pp.438-442.

8. Klopman, G. 1984. Artificial intelligence approach to structure activity studies. J. Amer. Chem. Soc., Vol.106, pp.7315-7321.

9. Klopman, G. 1992. MultiCASE 1. A hierarchical computer automated structure evaluation program, QSAR, Vol.11, pp.176-184.

10. Kramer, S., Pfahringer, B. and Helma, C. 1997. Mining for causes of cancer: Machine learning experiments at various levels of detail. In Proceedings of the Third International Conference on Knowledge Discovery and Data Mining (KDD-97), pp.223-226.

11. Mannila, H. and Toivonen, H. 1997. Levelwise search and borders of theories in knowledge discovery. Data Mining and Knowledge Discovery, Vol.1, No.3, pp.241258 .

12. Matsuda, T., Horiuchi, T., Motoda, H. and Washio, T. 2000. Extension of GraphBased Induction for General Graph Structured Data. In Proceedings of the Fourth Pacific-Asia Conference of Knowledge Discovery and Data Mining (PAKDD2000), pp.420-431.

13. Srinisavan, A., King, R.D., Muggleton, S.H. and Sternberg, M.J.E. 1997. The predictive toxicology evaluation challenge. In Proceedings of the Fifteenth International Joint Conference on Artificial Intelligence (IJCAI-97), pp.4-9.

14. Wang, K. and Liu, H. 1997. Schema discovery for semistructured data. In Proceedings of the Third International Conference on Knowledge Discovery and Data Mining (KDD-97), pp.271-274 